\title{
Simulation of pedestrian single lane movement by a biped model
}

\author{
Zhongyi Huang, ${ }^{1, *}$ Mohcine Chraibi, ${ }^{2, \dagger}$ and Weiguo Song ${ }^{1, \ddagger}$ \\ ${ }^{1}$ State key Laboratory of Fire Science, University of Science and Technology of China, Hefei 230027, China \\ ${ }^{2}$ Institute for Advanced Simulation, Forschungszentrum Jülich, 52425 Jülich, Germany
}

(Dated: November 1, 2018)

\begin{abstract}
In most existing pedestrian dynamic models, agents are modeled as sliding or jumping objects. As a kind of bipedal creature, however, this assumption makes it difficult to include some important human walking characteristics in the models, such as the periodicity of stepping, the adjustment of step frequency and step length, and the change of personal space within the same step cycle. To take these characteristics into consideration, a "Biped Model" is developed to simulate pedestrian locomotion by physical step rather than by time step. The model consists of two components: the stepping simulator and the adjustment of velocity for each step. Simulation results show that the velocity-density relations are consistent with the empirical data. Stable stop-and-go waves are simulated with a critical density. Furthermore, with the considering of biped movement, the lock-step phenomenon is reproduced. The model provides new possibilities to study crowd behavior while considering personal bipedal mechanics.
\end{abstract}

Keywords: pedestrian dynamics, Biped Model, foot movement, stop-and-go waves, lock-step phenomenon

\section{INTRODUCTION}

Simulation of pedestrian movement has been a hot topic in recent years, because of its extensive application in safety, building design, traffic facilities design, and robotics. Many two-dimensional (2D) models have been developed to simulate the movement with different approximations of the projection of pedestrian's shape. A circle is the most commonly used shape [1-4]. The diameter of the circle is of the same width as the shoulders. In cellular atuomata (CA) models, an agent is assigned a grid of the cell. Shapes of the cell are usually square [5-7] or hexagonal $[8,9]$. To make the space in CA more flexible, multigrid models $[10,11]$ are developed by assigning pedestrians in small $3 \times 3$ grids.

For the sake of more "accuracy", several researchers opted for different shapes to model pedestrian's volume exclusion. In Ref. [12], a pedestrian is represented by three intersecting circles. In Ref. [13], a spherocylindrical particle is adopted to naturally reproduce evacuations through narrow doors. Marroquin et al. [14] approximate the shape of a chest cross section of a human thorax by the concept of Minkowski's sum of a polygon with a disk. The "body ellipse" was suggested to be the best twodimensional approximation of the human body $[15,16]$. Based on this idea, a generalized centrifugal-force model which included an elliptical volume exclusion of humans was proposed by Chraibi et. al [17].

Although the above-mentioned models differ in precision and updating strategy, they still have something in common: pedestrians are integral and their movements are nonperiodic. Integral means a whole without elements have relative motions. Nonperiodic means no

\footnotetext{
* hzy1992@mail.ustc.edu.cn

$\dagger$ m.chraibi@fz-juelich.de

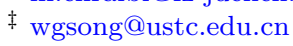

biomechanical cycles are included. In this paper, we refer to these integral and nonperiodic models as "particle models".

As a kind of bipedal creature, humans move forward by stepping alternately with the left and right foot. This kind of movement pattern results in some unique phenomena that can hardly be investigated by particle models: lock-step phenomenon in high density crowd, tumble and stampede, the effect of music and rhythm on individual or crowd behavior, and pedestrians can still move when there is no spare space for their bodies from the top view. We think the above phenomena mainly result from the following three characteristics of biped movement: At first, walking is a periodic movement, and the expected velocity is always stable within a step cycle. If the actual velocity differs largely from the expected velocity, i.e. pushed by others, stepping on others' feet or stepping with an unexpected drop, a pedestrian may fall down and a stampede accident may follow. Furthermore, pedestrians adjust their velocity by both step length and frequency. The walking behavior can be affected if one of them is influenced by external environment, e.g. rhythm [18]. Finally, volume exclusion of a pedestrian changes during a step because of the stretching of legs. This could be one reason for the lock-step phenomenon [19]: pedestrians squeeze the front leg into the hole left by the leg of the preceding pedestrian. For the same reason, pedestrians can still move even when there is no spare space for bodies while there is still room for feet. The former two features are also noticed by the works related to the optimal step model (OSM) [20, 21], in which pedestrians are simulated by periodically moving particles. Pedestrian movement in the OSM is natural because they have similar movement cycle and step length adaption behavior as humans. However, the model is rather simple since it does not consider the space change caused by bipedal movement during one step cycle.

To take these characteristics into consideration, a 
biped model is developed in this paper to simulate pedestrians by natural movements of two feet. A schematic of the model will be introduced in Section II. Two parts in the schematic, the stepping simulator and the step level velocity adjustment will be developed in Secs. III and IV respectively. Then results of the model are compared with experimental results in Section V. Finally, the conclusion is given in Sec. VI.

\section{MODEL SCHEMATIC}

In our model, the spatial state of pedestrian $i$ at time $t$ is denoted by the heels and toes of both feet:

$$
P_{i}(t)=\left[L_{i}^{h}(t), L_{i}^{t}(t), R_{i}^{h}(t), R_{i}^{t}(t)\right],
$$

where $L_{i}^{h}=[x, y]^{T}$ and $L_{i}^{t}=\left[x^{\prime}, y^{\prime}\right]^{T}$ are the coordinates of the heel and toe of the left foot. Based on $P_{i}$, the physical space $\left(s_{i}\right)$ of a pedestrian is defined as the polygon whose vertexes are the four points. Head position is the middle point of the two heels. Headway distance $\left(d_{i}\right)$ is the distance between head positions of two adjacent pedestrians (see Fig. 1(a)).

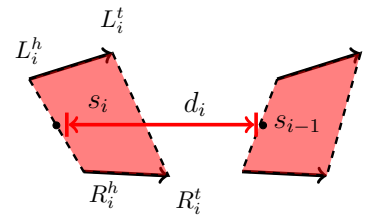

(a)

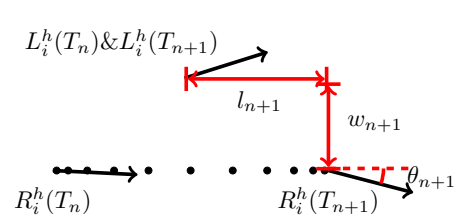

(b)
Fig. 1. Spatial structure of walking. (a)Definitions of spatial state $\left(P_{i}\right)$, head position and physical space $\left(s_{i}\right)$. (b)Definition of step width $(w)$, step length $(l)$ and foot open angle $(\theta)$.

According to Boulic et al. [22], a pedestrian has two states while walking: the duration of double support (both feet in contact with the ground) and the duration of balance (one foot in contact with the ground). In our model, we assume that one foot leaves the ground and the other foot touches the ground happen at the same time. That means that, during each step, there is only one moment at which both feet are on the ground. We refer to this moment as the stand moment. The n-th stand moment is labeled $T_{n}$. Based on this assumption, the temporal structure of the model is shown in Fig. 2, in which time is discretized by the time step $(\delta)$. Stand moments are rounded to the nearest time step. The time between $T_{n}$ and $T_{n+1}$ is the duration of a step $\left(\Delta_{n+1}\right)$. Except for stand moments, all the other time steps are balance moments.

Then spatial states of a step can be visualized in Fig. 1 (b). The black arrows are the feet at stand moments and the black points are the heels at balance moments. According to the definition of a stand moment, the black arrows are also footprints. To describe the relative position

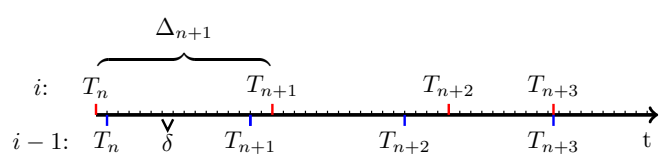

Fig. 2. Temporal structure of walking of the model.

relationship between footprints, three spatial quantities are defined. Supposing pedestrians are walking along the $\mathrm{x}$-axis in the single lane scenario discussed in this paper, the step length $\left(l_{n+1}\right)$ of the $(\mathrm{n}+1)$-th step is the distance between heels along the x-axis, step width $\left(w_{n+1}\right)$ is the distance between heels along the y-axis, and foot open angle $\left(\theta_{n+1}\right)$ is the angle between the front footprint and the $\mathrm{x}$-axis.

The main idea of the model is that pedestrians plan their movement by physical steps rather than by time steps. In the biped model, velocity is only planned when a step is finished (stand moments). While at balance moments, pedestrians just execute the planned step. If two adjacent pedestrians finish a step at the same time $\left(t=T_{n+3}\right.$ for both pedestrians in Fig. 2), the front pedestrian (referred to as "leader") will decide at first.

A schematic of the model is shown in Fig. 3, in which time increases in nature with the time step $(t=t+\delta)$. When a step of a pedestrian is finished $\left(t=T_{n}\right)$, velocity of the next step $\left(v_{n+1}\right)$ will be planned by the three updating processes proposed in Section IV. With $v_{n+1}$ we can solve the spatial states of the next step $\left(P_{i}(t), t \in\left(T_{n}, T_{n+1}\right]\right)$ by the stepping simulator developed in Section III. Note that the "Stepping simulator" is called by "Collision avoidance" in the process of velocity updating, as a result Section III (Stepping simulator) will be introduced before Section IV (Updating of velocity).

\section{STEPPING SIMULATOR}

\section{A. Generation of spatial state at stand moment}

According to the temporal structure shown in Fig. 2, time of the new stand moment is:

$$
T_{n+1}=T_{n}+\Delta_{n+1} .
$$

As the spatial structure shown in Fig 1 (b), spatial state of the new stand moment is ${ }^{1}$ :

$$
P_{i}\left(T_{n+1}\right)=\left[L_{i}^{h}\left(T_{n}\right), L_{i}^{t}\left(T_{n}\right), R_{i}^{h}\left(T_{n+1}\right), R_{i}^{t}\left(T_{n+1}\right)\right],
$$

\footnotetext{
${ }^{1} P_{i}\left(T_{n+1}\right)$ can be solved in a similar manner when the left foot is stepping forward from $T_{n}$ to $T_{n+1}$.
} 


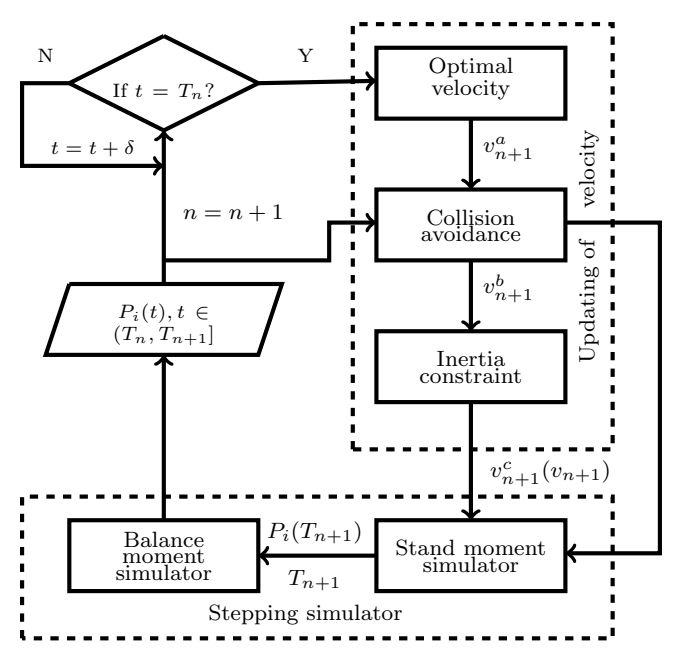

Fig. 3. Schematic of the biped model. The stand moment simulator refers to the generation of spatial state at stand moment described in Section III A. The balance moment simulator refers to the generation of a spatial state at the balance moment described in Section III B.

where

$$
\begin{aligned}
R_{i}^{h}\left(T_{n+1}\right) & =L_{i}^{h}\left(T_{n}\right)+\left[l_{n+1},-w_{n+1}\right]^{T}, \\
R_{i}^{t}\left(T_{n+1}\right) & =R_{i}^{h}\left(T_{n+1}\right)+f\left[\cos \theta_{n+1},-\sin \theta_{n+1}\right]^{T} .
\end{aligned}
$$

$f$ is the foot length of the pedestrian. According to Ilayperuma et. al [23], there is the following linear dependence between $f$ and human's height $(H)$ :

$$
f(H)=(H-0.79) / 3.59 .
$$

In Eqs. (3) and (4) we see that the left foot is static from $T_{n}$ to $T_{n+1}$. The heel position of the right foot $R_{i}^{h}\left(T_{n+1}\right)$ is calculated by $l_{n+1}$ and $w_{n+1}$ at first, then the toe position is solved by $R_{i}^{h}\left(T_{n+1}\right)$ and $\theta_{n+1}$. To summarize, $\Delta, l, w$ and $\theta$ are needed to generate the movement of a new stand moment. A global human walking model was proposed by Boulic et. al [22], in which the values of $l_{n+1}, w_{n+1}$ and $\theta_{n+1}$ are functions of the velocity $\left(v_{n+1}\right)$ of a step $^{2}$ :

$$
\begin{aligned}
l(v) / g & =0.637 \sqrt{v / g}, \\
w(v) / g & =0.02 v / g+0.05, \\
\theta(v) & =-1.4 v / g+8.5,
\end{aligned}
$$

where $g$ is the thigh height. According to Kreighbaum et al. [24] $g$ is about $53 \%$ of a pedestrian's height:

$$
g(H)=0.53 H
$$

\footnotetext{
${ }^{2}$ For simplicity, the subscripts of $\Delta, l, w, \theta$ and $v$ are omitted in the following discussion.
}

In the single lane scenario, the step duration $(\Delta)$ is determined when $l$ and $v$ are known:

$$
\Delta(v)=l(v) / v=0.637 \sqrt{g / v}
$$

In Ref. [22], the applicable velocity of Eqs. (6)-(10) is from $0.6 \mathrm{~m} / \mathrm{s}$ to $2.0 \mathrm{~m} / \mathrm{s}$ when $g=1 \mathrm{~m}$. Therefore these functions can not be applied in low velocity situations (such as $v=0.17 \mathrm{~m} / \mathrm{s}$ when $\rho=1.97 \mathrm{ped} / \mathrm{m}$ [19]). To validate these functions, especially in low velocity situation, controlled experiments were conducted. The schematic of the experimental set up is shown in Fig. 4 (a).

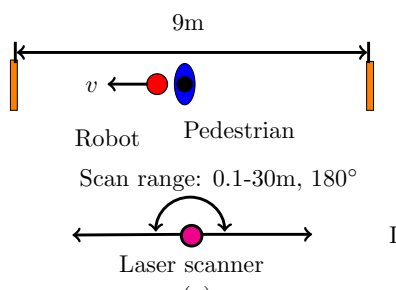

(a)

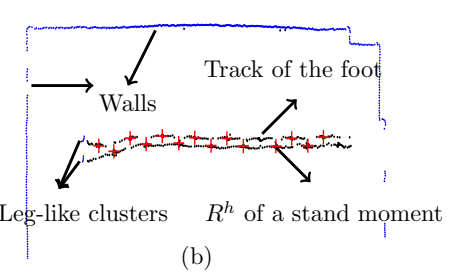

(b)
Fig. 4. (a) Schematic of experiments. (b) Results extracted from one experiment.

In each experiment, a pedestrian kept following a straight moving robot for about $9 \mathrm{~m}$ at a constant velocity. Velocities of the robot were $0.2,0.4,0.6,0.8,1.0$ and $1.2 \mathrm{~m} / \mathrm{s}$ respectively. Finally, the pedestrian walked at a normal velocity without following the robot. The experiment at each velocity was repeated four times. Six male and four female university students took part in the experiments. A laser range scanner (type: HOKUYO UTM-30LX) was set at a height of $0.15 \mathrm{~m}$ to record the ankle movement. With the method described in Appendix $\mathrm{A}$, the heel positions and the time of all stand moments could be extracted (see 4 (b)). With these data, Eqs. (6), (7) can be validated. The results are shown in Fig. 5.

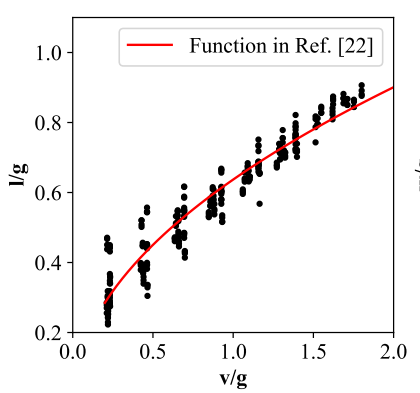

(a)

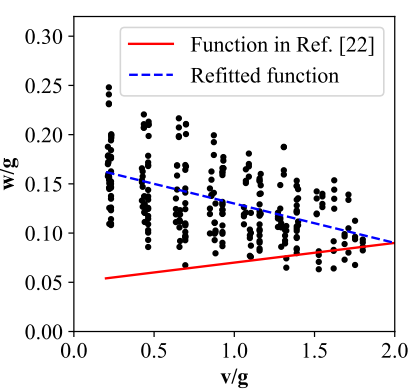

(b)
Fig. 5. Relationships obtained from robot following experiments. (a) Normalized step length $(l / g)$ as a function of normalized velocity $(v / g)$. (b) Normalized step width $(w / g)$ as a function of normalized velocity $(v / g)$. 
According to Fig. 5 , Eq. (6) has a good agreement with the experiments, while Eq. (7) shows a trend opposite that of the experiments. We refit the relation between $w$ and $v$ as follows:

$$
w(v) / g=-0.04 v / g+0.17 \text {. }
$$

The above relations are applicable when $v>0.2 \mathrm{~m} / \mathrm{s}$. Nevertheless in high density situations, some extreme slow steps may be adopted by pedestrians. According to Ref. [25], pedestrians continue to sway and shift their body weight from one leg to the other when the density is too high to force them almost to stop. Comparing to the dependence between velocity and step length, the increase in step duration is much more gradual with the decrease of velocity, and the largest duration of a step is about 1.2 s. But with Eq. (10), a step has a duration of $6.37 \mathrm{~s}$ when an extreme low velocity $(v=0.01 \mathrm{~m} / \mathrm{s})$ is planned. It is much larger than the maximum duration in experiments. To make the simulator more realistic at low velocity, we controlled the duration of a step by setting a cutoff time $C$ for $\Delta$ :

$$
\Delta(v)= \begin{cases}0.637 \sqrt{g / v} & 0.637 \sqrt{g / v} \leq C \\ C & 0.637 \sqrt{g / v}>C .\end{cases}
$$

Then the step-length function Eq. (6) should be adjusted accordingly to guarantee the velocity after the cut-off:

$$
l(v)=v \Delta(v) .
$$

Based on the discussion above, the time and spatial state of the next stand moment can be generated by the velocity with a wide range from 0 to about $2 \mathrm{~m} / \mathrm{s}$. Equations (8), (11) and (12) should first be computed to obtain $\theta, w$ and $\Delta$, then we calculate $l$ with $\Delta$ obtained from Eq. (13). Finally, we solve the new stand moment by using Eqs. (2)-(4).

\section{B. Generation of spatial state at balance moment}

At balance moments, the space occupied by a pedestrian is changing because of the swing of the foot. Hence spatial states at balance moments are necessary in the collision avoidance process which is introduced in Section IV B. The uniform accelerating model in Ref. [26] is adopted to solve for the heel position at balance moments, in which the swing foot first accelerates uniformly and then decelerates uniformly between two stand moments. In addition, we suppose that the foot opening angle changes uniformly at the same time. Based on the two assumptions, spatial states of the feet at balance moments between $P_{i}(t)$ and $P_{i}\left(T_{n+1}\right)$ in Fig. 1 (b) can be solved by ${ }^{3}$ :

\footnotetext{
3 By analogy it can be solved in a similar manner when the left foot is stepping forward.
}

$$
P_{i}(t)=\left[L_{i}^{h}\left(T_{n}\right), L_{i}^{t}\left(T_{n}\right), R_{i}^{h}(t), R_{i}^{t}(t)\right], t \in\left(T_{n}, T_{n+1}\right)
$$

where

$$
\begin{aligned}
& R_{i}^{h}(t)=\left\{\begin{array}{c}
R_{i}^{h}\left(T_{n}\right)+2 t^{\prime 2}\left(R_{i}^{h}\left(T_{n+1}\right)-R_{i}^{h}\left(T_{n}\right)\right), \\
0<t^{\prime} \leq 1 / 2 \\
R_{i}^{h}\left(T_{n+1}\right)-2\left(1-t^{\prime}\right)^{2}\left(R_{i}^{h}\left(T_{n+1}\right)-R_{i}^{h}\left(T_{n}\right)\right), \\
1 / 2<t^{\prime} \leq 1
\end{array}\right. \\
& t^{\prime}=\left(t-T_{n}\right) /\left(T_{n+1}-T_{n}\right) \\
& R_{i}^{t}(t)=R_{i}^{h}(t)+f\left[\cos \theta^{\prime},-\sin \theta^{\prime}\right] \\
& \theta^{\prime}=\theta_{n}+\left(\theta_{n+1}-\theta_{n}\right) t^{\prime} .
\end{aligned}
$$

\section{UPDATING OF VELOCITY}

With the stepping simulator developed in Section III, the foot movement of a new step $\left(P_{i}(t), t \in\left(T_{n}, T_{n+1}\right]\right)$ can be simulated with a given velocity $v_{n+1}$. In this section, we discuss how to update $v_{n+1}$ according to the bipedal movements.

\section{A. Optimal velocity}

At first, a pedestrian makes a temporary decision considering the headway distance $(d)$, which is called an optimal velocity process. In the experiment presented by Jelić et al [27], the instantaneous individual measurements were adopted: Measuring the instantaneous velocity and the headway distance at each frame. When a pedestrian in our model makes a decision, she faces a similar situation with the instantaneous measurement: Determining the velocity of next step by the instantaneous headway distance. Instantaneous velocity as a function of headway distance in Ref. [27] is shown in Fig. 6.

In Ref. [27] the authors distinguished the dependence into three regimes: a free regime $(d>3 \mathrm{~m})$, a weakly constrained regime $(1.1 \mathrm{~m}<d \leq 3 \mathrm{~m})$ and a strongly constrained regime $(d \leq 1.1 \mathrm{~m})$. Therefore, we fit the relation with a piecewise function:

$$
v_{n+1}^{a}(d)=\left\{\begin{array}{c}
\tanh (2.4 *(d-0.85) / 2+0.5) * 1.16 \\
d \leq 1.1 \\
F(0.53 d-0.58)-0.47 d+1.41 \\
F, d>3,
\end{array}\right.
$$

where $F$ is the free walking velocity of the pedestrian. 


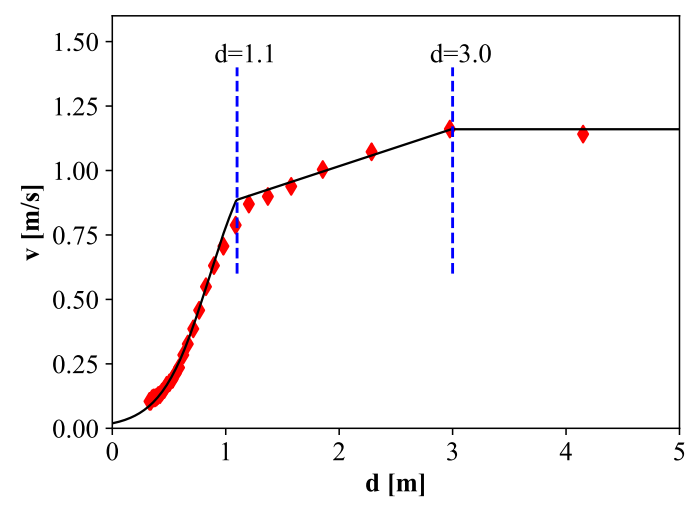

Fig. 6. Velocity as a function of headway distance. Red diamonds are data from Ref. [27], and the black lines are the fitting results.

\section{B. Collision avoidance}

According to Ref. [3], an optimal velocity function based model with a high updating frequency $(100 \mathrm{~Hz}$ in Ref. [3]) is collision free if velocity is $0 \mathrm{~m} / \mathrm{s}$ when the minimal spacing in front is $0 \mathrm{~m}$. However, according to the definition of headway distance, $d$ in Eq. (16) is larger than the minimal space, i.e. the distance between the backward heel of the leader and forward toe of the follower. Besides, in the biped model the updating frequency is uncertain, and could be quite low when a slow step is adopted $(0.83 \mathrm{~Hz}$ when $\Delta=1.2 \mathrm{~s})$. Furthermore the space of a pedestrian is dynamic for all time steps in the biped model, which also increases the possibility of collision. As a result, with the velocity obtained by Eq. (16), a collision in the next step can not be excluded. Collision avoidance is necessary after the optimal velocity process.

In Section II we describe the 2D space occupied by a pedestrian as a physical space $s$ (see Fig. 1(a)). However, if we use $s$ to conduct collision avoidance directly, pedestrians will be aggressive: Stepping close to the leader's feet as long as no collision happens. If the leader decelerates during this period, the follower may step on the leader's foot. An extension coefficient $E$ is introduced to guarantee a safety margin. Space for collision avoidance is obtained by extending $s$ along the $x$ and the $y$ axis by $E$. We outline the collision avoidance process in Algorithm 1, where we see that the spatial states of the next step of pedestrian $i$ are first generated by the stepping simulator with the result of the optimal velocity function. Next, a while loop is conducted to find a velocity which can pass the collision test. The collision test process is shown in Fig. 7, in which the model checks if the two polygons of the follower and the leader overlap during the oncoming step (from $T_{n}$ to $T_{n+1}$ ) with the method described in Appendix B. If a collision happens $\left(t=T_{n}+8 \delta\right.$ in Fig. $\left.7(\mathrm{a})\right)$, the velocity is rejected in the test. We reduce the velocity by $0.05 \mathrm{~m} / \mathrm{s}$ each time until we obtain a velocity that can pass the test. In Fig. 7 (b), no collision happens when the velocity is reduced to $0.27 \mathrm{~m} / \mathrm{s}$, which is the result of the collision avoidance process: $v_{n+1}^{b}=0.27 \mathrm{~m} / \mathrm{s}$.

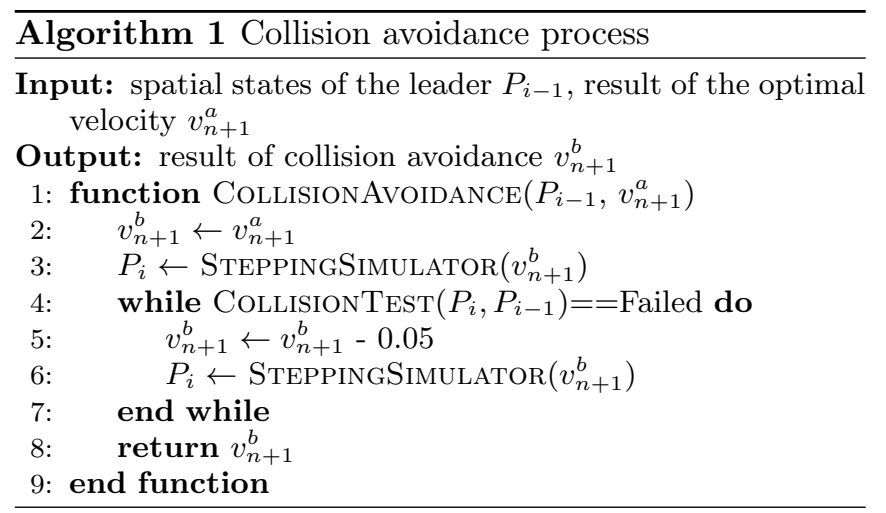

\section{Inertia constraint}

Due to inertia, there is a limitation of velocity change $(L)$ between two steps. We estimate the value of $L$ by free accelerated walking. For example, when $L=0.4 \mathrm{~m} / \mathrm{s}$, $F=1.29 \mathrm{~m} / \mathrm{s}$ four steps are needed to accelerate to $F$, i.e. $v_{1}=0.4 \mathrm{~m} / \mathrm{s}, v_{2}=0.8 \mathrm{~m} / \mathrm{s}, v_{3}=1.2 \mathrm{~m} / \mathrm{s}, v_{4}=1.29 \mathrm{~m} / \mathrm{s}$. The trajectory of the head position within the four steps is the free acceleration trajectory of the the biped model. According to the experimental study in Ref. [28], the acceleration process can be described as a nonuniformly accelerating movement. The acceleration at a specific $v$ is $a=(F-v) / \tau$, where $\tau=0.5 \mathrm{~s}$ is the relaxation time. With the function, we can obtain the free acceleration trajectory of experiment. Five trajectories are simulated in the biped model by setting $L=0.4,0.6,0.8,1.0$ and $1.2 \mathrm{~m} / \mathrm{s}$ respectively. By comparing the length of the five trajectories with the length of experiment trajectory, we find that the deviation of length is minimal when $L=0.8$ $\mathrm{m} / \mathrm{s}$. As a result, $0.8 \mathrm{~m} / \mathrm{s}$ is the value of $L$ in the Biped Model. Restricting the result of collision avoidance by $L$ is the final velocity of the new step:

$$
v_{n+1}=v_{n+1}^{c}= \begin{cases}v_{n}-L & v_{n+1}^{b}-v_{n}<-L \\ v_{n+1}^{b} & \left|v_{n+1}^{b}-v_{n}\right| \leq L \\ v_{n}+L & v_{n+1}^{b}-v_{n}>L\end{cases}
$$

\section{RESULTS}

With the stepping simulator and the velocity updating method, biped movement can be simulated in the single lane scenario. In this section, we evaluate the model by comparing the simulation results with experiments. Descriptions and values of the parameters used 


\section{$T_{n} \quad T_{n}+\delta$ \\ " " " " "}

(a): $v_{n+1}=0.32 \mathrm{~m} / \mathrm{s}$, failure

\section{$T_{n} \quad T_{n}+\delta$

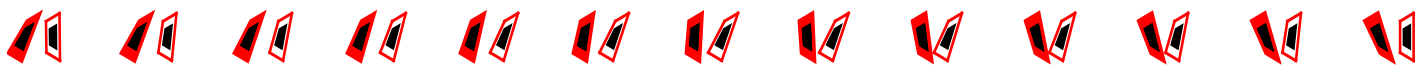

(b): $v_{n+1}=0.27 \mathrm{~m} / \mathrm{s}$, success

Fig. 7. Collision test process. Black core is $s$, filled and empty red polygons are the extended space of the follower and the leader respectively. (a) When $v_{n+1}=0.32 \mathrm{~m} / \mathrm{s}$, collision happens at $t=T_{n}+8 \delta$. (b) When $v_{n+1}=0.27$ $\mathrm{m} / \mathrm{s}$, no collision happens in the new step.

in the model are listed in Table I. To simulate the differences between individuals, the parameters are normally distributed. The values of $C, F$ and $L$ are already specified in Secs. III and IV. The value of $E$ is discussed later. Except for the five parameters listed in the table, all other definitions are dependent variables.

TABLE I: Descriptions and values of parameters in Biped Model. $\mathrm{N}(\mu, \sigma)$ indicates that the parameter is normally distributed in a crowd with mean value $(\mu)$ and standard deviation $(\sigma)$.

\begin{tabular}{llll}
\hline Parameter & Description & Value & Unit \\
\hline$H$ & height of pedestrian & $\mathrm{N}(1.70,0.05)$ & $\mathrm{m}$ \\
$C$ & max duration of a step & $\mathrm{N}(1.20,0.05)$ & - \\
$F$ & free walking velocity & $\mathrm{N}(1.29,0.05)$ & $\mathrm{m} / \mathrm{s}$ \\
$E$ & extension coefficient of $s$ & To be determined & - \\
$L$ & limitation of velocity change & $\mathrm{N}(0.80,0.03)$ & $\mathrm{m} / \mathrm{s}$ \\
\hline
\end{tabular}

The scenario of the following simulations consists of uniformly distributed pedestrians walking in a $10 \mathrm{~m}$ corridor with periodic boundary conditions. The time step $\delta$ is $0.04 \mathrm{~s}$. All pedestrians stand still at the beginning and a pedestrian is chosen randomly to make the first step with a random foot. Others make the first step by trying with two feet, and choosing the foot to make velocity of the first step larger. If the velocities are the same in the two trails, the foot to conduct the first step is chosen randomly. Each simulation lasts 4000 time steps (160 s). Nineteen simulations with densities ranging from 0.2 to $2.0 \mathrm{ped} / \mathrm{m}$ are conducted five times each.

\section{A. Fundamental diagram}

During the simulation we find that the velocity is not stable at the beginning of a simulation. Hence the average velocity of each simulation is computed after $80 \mathrm{~s}$. The average value $(\mu)$ and standard deviation $(\sigma)$ of the five repeated simulations at each density are compared in Fig. 8 with the experimental results of Ref. [27] .

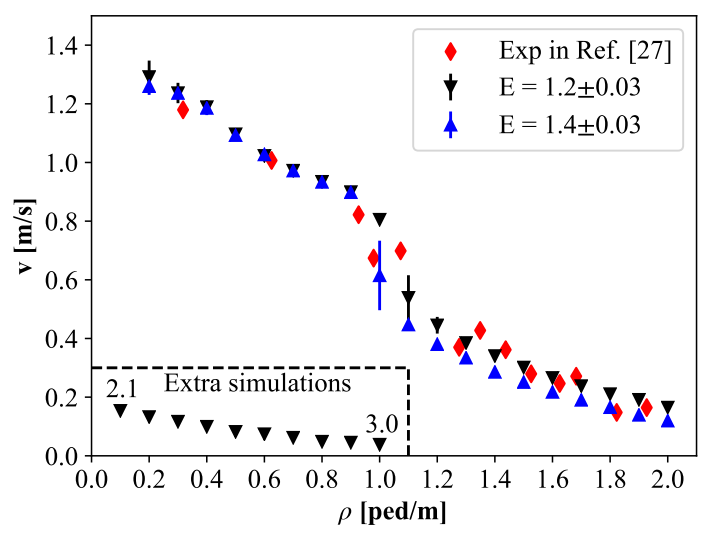

Fig. 8. Fundamental diagram of experiments and simulations. The points are $\mu$ of velocities in five repeated simulations, and the vertical lines are $\sigma$ of velocities.

From the figure we see that velocities with different $E$ are nearly the same when density is $<1.0 \mathrm{ped} / \mathrm{m}$. However, when density grows, $E=1.2 \pm 0.03$ leads to a better fit. Velocities with $E=1.4 \pm 0.03$ in the simulation are always less than in experiments. This can be explained by the fact that, with larger $E$, pedestrians need a larger walking space. Considering the good agreements between experiments and simulations, $1.2 \pm 0.03$ is set as the value of $E$. Benefiting from the accurate description of space, pedestrian movement can be simulated at quite a high density in the biped model. Extra simulations are conducted with $E=1.2 \pm 0.03$ to test the maximal applicable density of the model. As the black points shown in left bottom of Fig. 8, the average velocity declines gradually and keeps a nonzero value until $3.0 \mathrm{ped} / \mathrm{m}$. In most particle models, the diameter of a pedestrian is usually between $0.4 \mathrm{~m}$ and $0.5 \mathrm{~m}$. This means that, at this density, pedestrians already overlap with each other. Addition- 
ally, we find that there is a fast decline region (density from 1.0 to $1.2 \mathrm{ped} / \mathrm{m}$ ) in both experiments and simulations.

\section{B. Stop-and-go wave}

Stop-and-go waves have been observed in a lot of pedestrian dynamic experiments [25, 29, 30]. By analyzing these experiments, some researchers find that there is a critical density for this phenomenon in the single-lane scenario. In data of German soldiers [30], stopping was first observed during runs with 45 pedestrians in a $26 \mathrm{~m}$ corridor $(1.73 \mathrm{ped} / \mathrm{m})$. In French experiments [25], stopand-go waves arise when the density is larger than 1.20 $\mathrm{ped} / \mathrm{m}$. To investigate the ability of the biped model to reproduce realistic stop-and-go waves, pedestrians' positions as a function of time in three simulations are shown in Fig. 9. At the same time, $\mu$ and $\sigma$ of all pedestrians' velocities are also recorded every two seconds.

As shown in Fig. 9, stable stop-and-go waves appear when density $\geq 1.1 \mathrm{ped} / \mathrm{m}$. The critical density in the simulation is close to that in Ref. [25]. Furthermore, we observe that small fluctuations are magnified over time at the beginning. At the same time, $\mu$ decreases and $\sigma$ increases. After a period of time, a stable jam emerges, and both $\mu$ and $\sigma$ of velocity become stable. Generally speaking, shorter time is needed to reach the stable state in higher density. Then the findings mentioned in Section V A can be explained: The formation of stop-and-go waves give rise to the fast decline zone.

\section{Lock-step phenomenon}

The lock-step phenomenon was noticed in Ref. [19], which can be described as pedestrians squeezing the front leg at the gap left by the leg of the leader for the full use of space at high density. This phenomenon indicates that the space a pedestrian takes up changes not only with velocity, but also with time within a step, and pedestrians can optimize their use of the space. To investigate if this phenomenon can be reproduced in our model, we use a quantitative method proposed in Ref. [25]. $\Phi$ is defined in this method to measure the synchronization among pedestrians:

$$
\Phi=2 \pi\left(t_{i}-t_{i-1}\right) / \Gamma_{i-1}
$$

where $t_{i}$ is the start time of the follower's walking cycle (a walking cycle contains two steps), and $\Gamma_{i-1}$ is the duration of the leader's walking cycle. According to the definition, if $\Phi=0$, the two adjacent pedestrians take the same foot at the same time (synchronization). In Ref. $[25], \Phi$ was grouped by instantaneous density ( $\rho$, inverse of the instantaneous headway distance). They observed that at large densities (beyond $1.25 \mathrm{ped} / \mathrm{m}$ ), there was a peak around the phase $\Phi=0$, which indicated the existence of the lock-step phenomenon (see Fig. 10, black dots). By using the same measurement, the result of simulations are also shown in Fig. 10.

According to the figure, a peak at $\Phi=0$ only appears when $\rho>1.5 \mathrm{ped} / \mathrm{m}$ in simulation. With increasing simulation time, the distributions of $\Phi$ are stable in Fig. 10(a), while the peak becomes lower in Fig. 10(b). This means that, in the biped model, the lock-step phenomenon only appears when $\rho>1.5 \mathrm{ped} / \mathrm{m}$ and the phenomenon disappears gradually with increasing simulation time. The fading of lock steps in a simulation can also be observed in Fig. 11, in which the first steps of most pedestrians are lock steps. As the simulation time increases, less lock steps are observed. This can be explained by the fact that, in the biped model, step level interactions are simulated by collision avoidance, and collisions seldom happen when $\rho \leq 1.5 \mathrm{ped} / \mathrm{m}$. As time passes, stop-andgo waves arise, which affects the lock-step mechanism. Pedestrians in the model are unable to adjust their step frequency to catch the leader's step while leaving a jam. As a result, the peak at $\Phi=0$ becomes lower.

\section{DISCUSSION AND OUTLOOK}

Modeling of pedestrian movement was divided by a top-down approach into strategic, tactical and operational levels [31]. Seitz et. al [21] pointed out that an inaccurate model of lower level corrupted the results on higher levels. In this paper we provide a realistic model at the underlying level. To guarantee the accuracy, most processes in the biped model are based on experiments data: Generating of stand moment movement is based on the empirical equations in Ref. [22], studies of step frequency in Ref. [25], and our robot following experiments. The swing movement model [26] is also derived from experiments. Updating of velocity is based on the instantaneous fundamental diagram in Ref. [27] and the accelerating studies in Ref. [28].

With these experimental data, natural foot movements are simulated and the characteristics mentioned in Sec. I are included: Pedestrian movement is periodic, pedestrians adjust both step frequency and step length, physical space varies during a step cycle. With the collision avoidance process, the possibility of overlap is low even when pedestrians make decisions at low frequency. If overlap still happens after the collision avoidance, that indicates that the leader may slow down sharply. The follower can not react in time, and finally step on the leader's heel. That could induce a tumble during evacuation. Meanwhile, pedestrians in the biped model can move at quite high densities (3 ped $/ \mathrm{m}$ described in Sec. V A) as long as there is space for a foot.

Although several phenomena has been reproduced, some discrepancies with experiments can be observed: no lock-steps appear when the density is between 1.25 and $1.50 \mathrm{ped} / \mathrm{m}$, and stop-and-go waves arise earlier than that 

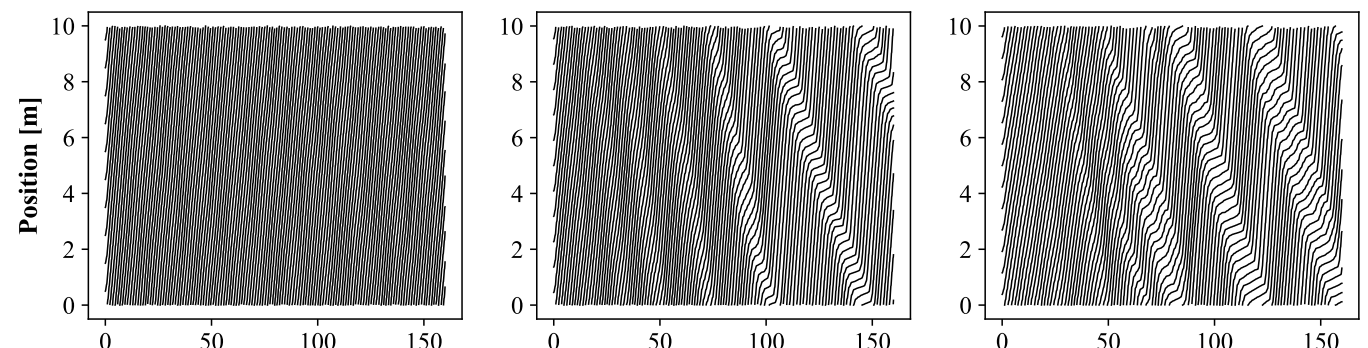

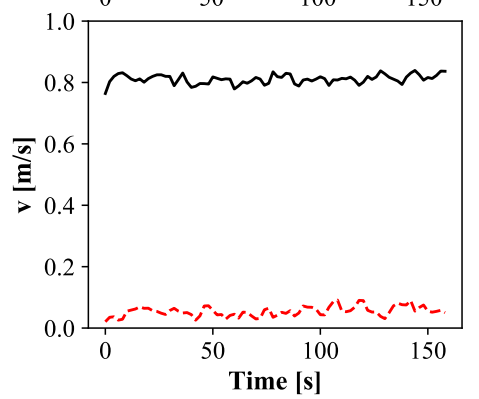

(a)

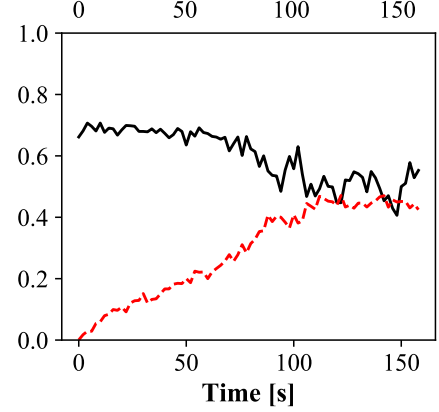

(b)

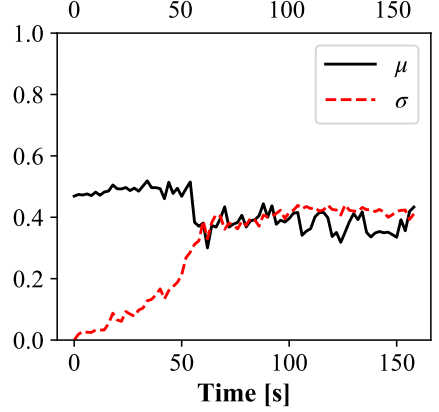

(c)

Fig. 9. Position, $\mu$ and $\sigma$ of velocity at different densities. (a) $1.0 \mathrm{ped} / \mathrm{m}$. (b) $1.1 \mathrm{ped} / \mathrm{m}$. (c) $1.3 \mathrm{ped} / \mathrm{m}$.

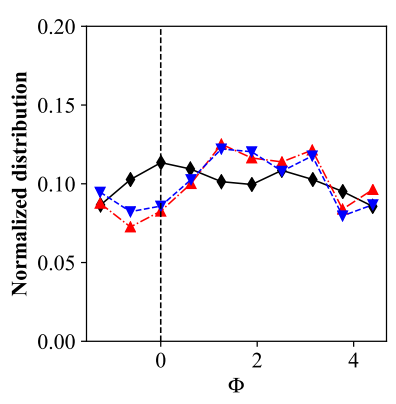

(a) (b)

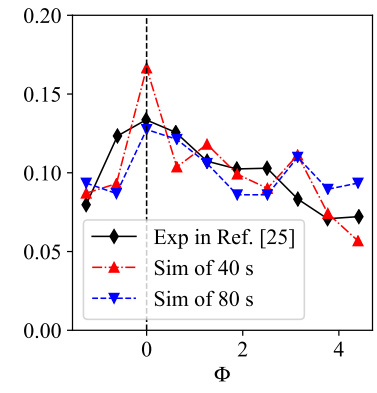

Fig. 10. Normalized distributions of $\Phi$. (a) $1.25 \mathrm{ped} / \mathrm{m}<\rho \leq 1.50 \mathrm{ped} / \mathrm{m}$. (b) $\rho>1.50 \mathrm{ped} / \mathrm{m}$.

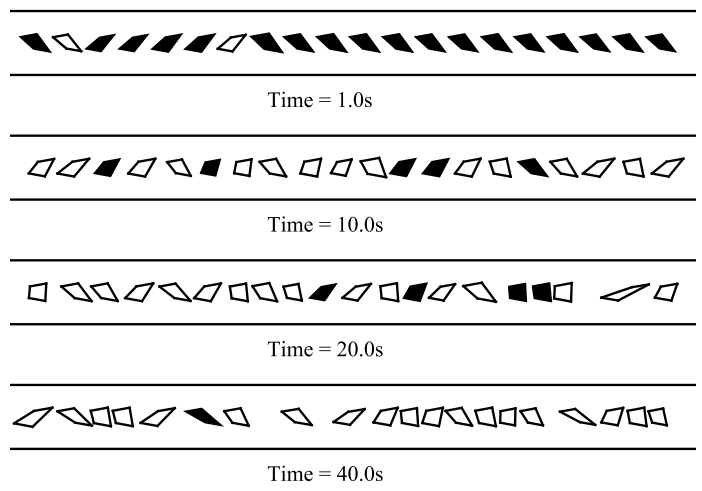

Fig. 11. Evolution of lock-step when density $=2.0$ ped $/ \mathrm{m}$. Filled polygons are lock-steps and empty polygons are non-lock steps. observed in some experiments. A possible explanation is that pedestrians in this density regime may have other step level considerations, e.g. the adjustment of step frequency according to the phase of the leader, which could have a deep impact on the lock-step phenomenon and stop-and-go waves. This consideration can be clarified through further experiments in future works. Furthermore, we strive to generalize the biped model to $2 \mathrm{D}$ scenarios.

\section{ACKNOWLEDGMENTS}

The authors would like to thank the Key Research and Development Program (2016YFC0802500) for support. Zhongyi Huang acknowledges funding support from the China Scholarship6 Council . Mohcine Chraibi acknowledges funding support from the Visiting Professor International project at the University of Science and Technology of China (Grant No. 2017B VR40).

\section{APPENDIX A: EXTRACTING BOTTOM POINTS FROM LASER RADAR DATA}

In each scan of the laser scanner, the raw data of both moving objects (e.g. pedestrians) and static objects (e.g. walls) can be collected (see blue points in Fig. 3 (b). At first, the static points are removed based on the distance histogram at each scanning angle [32]. Second, moving points are clustered: If the distance between two neighboring sample points is less than the threshold $(5 \mathrm{~cm})$, they will be classified as the same cluster. If the maximal distance between points in the cluster is similar to 
the diameter of the leg $(10-18 \mathrm{~cm})$, it will be regarded as a leg-like cluster. Centers of all leg-like clusters during the whole experiment are demonstrated by black points in the figure, according to which the black points are dense when the leg is during the support duration. Computing the local density around each black point, the point with the maximal local density is marked by the red crosses, which is also the approximate position of the heel.

\section{APPENDIX B: DETECTING OVERLAP BETWEEN TWO POLYGONS}

If any vertex in either polygon is inside the other one, the two polygons overlap. The judgment of whether a point is in a polygon is based on Franklin's PNPLOY algorithm [33]: Making a ray from the point to any direction, if the number of intersections between the ray and the edges of the polygon is even, the point is inside the polygon. Otherwise it is outside.
[1] D. Helbing and P. Molnar, Physical review E 51, 4282 (1995).

[2] W. Yu, R. Chen, L. Dong, and S. Dai, Physical Review E 72, 026112 (2005).

[3] A. Tordeux, M. Chraibi, and A. Seyfried, in Traffic and Granular Flow'15 (Springer, Cham, 2016) pp. 225-232.

[4] Y. Xiao, Z. Gao, Y. Qu, and X. Li, Transportation research part C: emerging technologies 68, 566 (2016).

[5] J. Ma, W.-g. Song, J. Zhang, S.-m. Lo, and G.-x. Liao, Physica A: Statistical Mechanics and its Applications 389, 2101 (2010).

[6] V. J. Blue and J. L. Adler, Transportation Research Part B: Methodological 35, 293 (2001).

[7] K. Nishinari, A. Kirchner, A. Namazi, and A. Schadschneider, IEICE Transactions on information and systems 87, 726 (2004).

[8] B. Leng, J. Wang, W. Zhao, and Z. Xiong, Physica A: Statistical Mechanics and its Applications 402, 119 (2014).

[9] M. Davidich, F. Geiss, H. G. Mayer, A. Pfaffinger, and C. Royer, Transportation Research Part C: Emerging Technologies 37, 210 (2013).

[10] W. Song, X. Xu, B.-H. Wang, and S. Ni, Physica A: Statistical Mechanics and its Applications 363, 492 (2006).

[11] S. Cao, W. Song, W. Lv, and Z. Fang, Physica A: Statistical Mechanics and its Applications 436, 45 (2015).

[12] P. A. Langston, R. Masling, and B. N. Asmar, Safety Science 44, 395 (2006).

[13] R. Hidalgo, D. Parisi, and I. Zuriguel, Physical Review E 95, 042319 (2017).

[14] F. Alonso-Marroquin, J. Busch, C. Chiew, C. Lozano, and Á. Ramírez-Gómez, Physical Review E 90, 063305 (2014).

[15] J. J. Fruin, Pedestrian planning and design (New York: Metropolitan Association of Urban Designers and Environmental Planners, New York, 1971).

[16] J. L. Pauls, in Conference Proceedings of the 3rd International Symposium on Human Behaviour in Fire (London, 2004).

[17] M. Chraibi, A. Seyfried, and A. Schadschneider, Physical Review E 82, 046111 (2010).

[18] D. Yanagisawa, A. Tomoeda, and K. Nishinari, Physical Review E 85, 016111 (2012).
[19] A. Seyfried, B. Steffen, W. Klingsch, and M. Boltes, Journal of Statistical Mechanics: Theory and Experiment 2005, P10002 (2005).

[20] I. von Sivers and G. Köster, Transportation Research Part B: Methodological 74, 104 (2015).

[21] M. J. Seitz and G. Köster, Physical Review E 86, 046108 (2012).

[22] R. Boulic, N. M. Thalmann, and D. Thalmann, The visual computer 6, 344 (1990).

[23] I. Ilayperuma, B. Nanayakkara, and K. Palahepitiya, Galle Medical Journal 13, 6 (2008).

[24] E. Kreighbaum and K. Barthels, "Biomechanics-a qualitative approach for studying human movement," (1996).

[25] A. Jelić, C. Appert-Rolland, S. Lemercier, and J. Pettré, Physical Review E 86, 046111 (2012).

[26] W. Chung, H. Kim, Y. Yoo, C.-B. Moon, and J. Park, IEEE transactions on industrial electronics 59, 3156 (2012).

[27] A. Jelić, C. Appert-Rolland, S. Lemercier, and J. Pettré, Physical Review E 85, 036111 (2012).

[28] M. Moussaïd, D. Helbing, S. Garnier, A. Johansson, M. Combe, and G. Theraulaz, Proceedings of the Royal Society of London B: Biological Sciences 276, 2755 (2009).

[29] A. Schadschneider, W. Klingsch, H. Klüpfel, T. Kretz, C. Rogsch, and A. Seyfried, in Extreme Environmental Events (Springer, New York, NK, 2011) pp. 517-550.

[30] A. Seyfried, M. Boltes, J. Kähler, W. Klingsch, A. Portz, T. Rupprecht, A. Schadschneider, B. Steffen, and A. Winkens, in Pedestrian and Evacuation Dynamics 2008 (Springer, Berlin, Heidelberg, 2010) pp. 145-156.

[31] S. P. Hoogendoorn and P. H. Bovy, Transportation Research Part B: Methodological 38, 169 (2004).

[32] X. Shao, H. Zhao, K. Nakamura, K. Katabira, R. Shibasaki, and Y. Nakagawa, in Intelligent Robots and Systems, 200\%. IROS 200\%. IEEE/RSJ International Conference on (IEEE, 2007) pp. 2174-2179.

[33] W. R. Franklin, Web site: http://www. ecse. rpi. edu/Homepages/wrf/Research/Short_Notes/pnpoly. html (2006). 